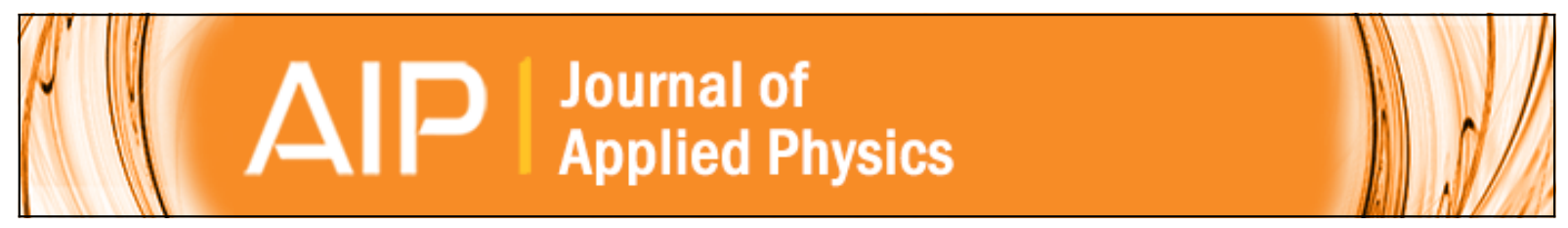

\title{
A quasi(unidirectional) Tellegen gyrator
}

Junyi Zhai, Jiefang Li, Shuxiang Dong, D. Viehland, and M. I. Bichurin

Citation: Journal of Applied Physics 100, 124509 (2006); doi: 10.1063/1.2402967

View online: http://dx.doi.org/10.1063/1.2402967

View Table of Contents: http://scitation.aip.org/content/aip/journal/jap/100/12?ver=pdfcov

Published by the AIP Publishing

\section{Articles you may be interested in}

The energy transfer between the ports of an implemented gyrator using LM13700 operational transconductance amplifier

Rev. Sci. Instrum. 83, 114702 (2012); 10.1063/1.4766332

Introduction to Gyrator Transform

AIP Conf. Proc. 949, 256 (2007); 10.1063/1.2812305

Radius of Gyration of a Sphere and a Barrel

Phys. Teach. 44, 222 (2006); 10.1119/1.2186232

Unidirectional magnetostrictive Terfenol/epoxy composite

Appl. Phys. Lett. 84, 2130 (2004); 10.1063/1.1688449

Shock response of a unidirectional composite at various orientation of fibers

AIP Conf. Proc. 429, 545 (1998); 10.1063/1.55563

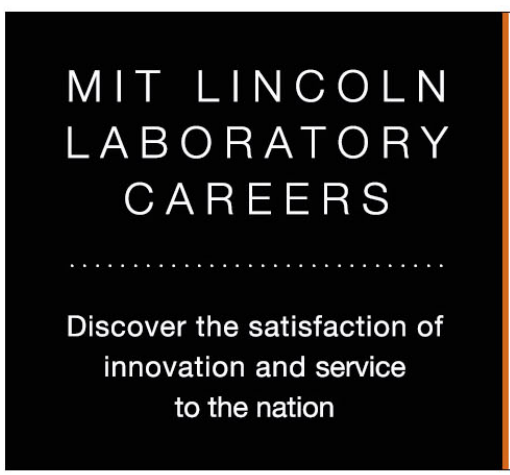
- Space Control
- Air \& Missile Defense
- Communications Systems
\& Cyber Security
- Intelligence, Surveillance and
Reconnaissance Systems

- Advanced
Electronics
- Tactical Systems
- Homeland
Protection
- Air Traffic Control

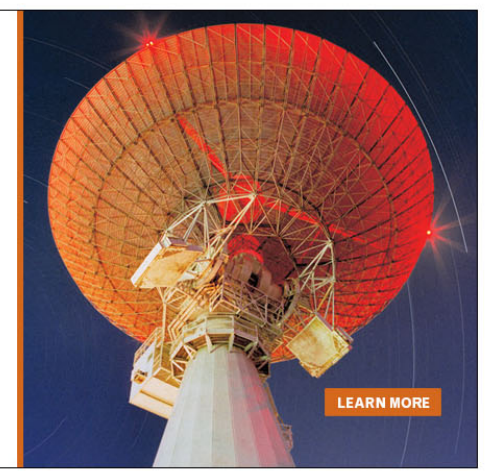




\section{A quasi(unidirectional) Tellegen gyrator}

Junyi Zhai, ${ }^{a}$ Jiefang Li, Shuxiang Dong, and D. Viehland

Department of Materials Science and Engineering, Virginia Tech, Blacksburg, Virginia 24061

M. I. Bichurin

Institute of Electronic and Informative Systems, Novgorod State University, 173003 Veliky Novgorod, Russia

(Received 5 September 2006; accepted 13 October 2006; published online 26 December 2006)

We show that magnetoelectric (ME) laminate composites have characteristics of a previously conjectured, but unfound, fifth network circuit element—-the Tellegen gyrator. Our findings establish that ME laminate composites (i) are nonreciprocal electrical elements, (ii) have significant nondissipative $I-V$ conversion near a resonance frequency, and (iii) act like a unidirectional gyrator, i.e., an ideal Tellegen gyrator connected with a capacitor on one side and an inductor on the other.

(C) 2006 American Institute of Physics. [DOI: 10.1063/1.2402967]

\section{INTRODUCTION}

In 1948, ${ }^{1}$ Tellegen of Philips Research Laboratories published seminal work on classic passive network elements, where he theorized that an additional network element based on magnetoelectric (ME) interactions might exist that he designated the gyrator. An ideal gyrator would be unique with respect to the other known four network elementscapacitor, resistor, inductor, and transformer-in that it would not comply with reciprocity but rather would be nonreciprocal. Well-known microwave gyrators which work on the Faraday effect in ferrites ${ }^{2}$ use another operational principle. However, over the course of many years, the notion/ hope of realizing a true passive network component with large gyration effects at lower frequencies has fallen into obscurity.

A four-pole circuit is shown in Fig. 1(a), the relations between the voltages and the currents can be expressed as

$$
\begin{aligned}
& V_{1}=Z_{11} I_{1}+Z_{12} I_{2}, \\
& V_{2}=Z_{21} I_{1}+Z_{22} I_{2},
\end{aligned}
$$

where $V$ is the voltage, $I$ the current, and $Z$ the impedance (which are functions of frequency). When $Z_{12}=-Z_{21}$ and $Z_{11}=Z_{22}=0$, the equations of (1) simplify to

$$
\begin{aligned}
& V_{1}=-\alpha I_{2}, \\
& V_{2}=\alpha I_{1},
\end{aligned}
$$

where $\alpha$ is a conversion coefficient between voltage and current. If a four-pole device obeyed this nonreciprocal relation, it would be an ideal gyrator as defined by Tellegen. ${ }^{1} \mathrm{He}$ conjectured that a media with both magnetization $\left(M_{S}\right)$ and polarization $\left(P_{S}\right)$ phases could be used to construct such a gyrator; however, at that time, ferromagnetoelectric materials were unknown.

Since Tellegen's time, magnetoelectric (ME) materials have been found and extensively investigated, as evidenced in reviews. ${ }^{3}$ The ME effect is a dielectric polarization change $\left(\Delta P_{S}\right)$ induced by a magnetic field $(H)$, or conversely a mag-

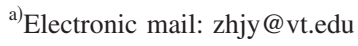

netization change $\left(\Delta M_{S}\right)$ induced by external electric field $(E)$. Unfortunately, the intrinsic ME effects in single phase materials are quite small. However, recently, we have reported giant ME effects in laminate composites of magnetostrictive and piezoelectric layers; ${ }^{4-8}$ however, we had not previously realized the existence or importance of gyration.

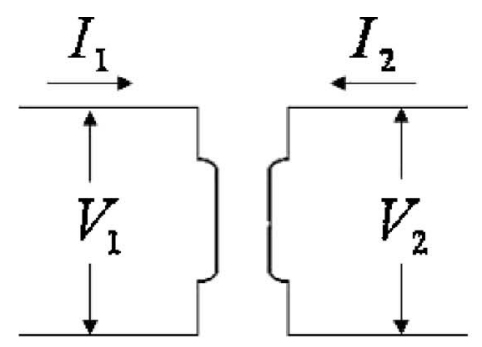

(a)

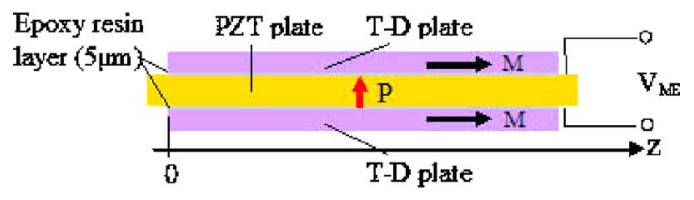

(b)

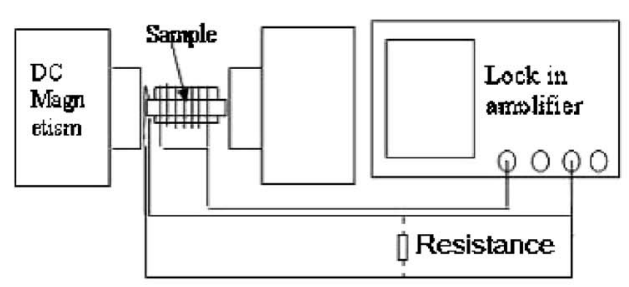

(c)

FIG. 1. (Color online) (a) Gyrator equivalent circuit; (b) illustration of longitudinal-transverse or $\mathrm{L}-\mathrm{T}$ mode of a magnetoelectric laminate composite consisting of longitudinally poled $\mathrm{Pb}\left(\mathrm{Zr}_{X} \mathrm{Ti}_{1-X}\right) \mathrm{O}_{3}$ layer sandwiched between two longitudinally magnetized Terfenol-D layers, epoxied together with a thin insulating resin layer; and (c) schematic illustrating the experiment setup. 
Here, we conclusively demonstrate the gyrator capabilities of composites consisting of a $\mathrm{Pb}\left(\mathrm{Zr}_{X} \mathrm{Ti}_{1-X}\right) \mathrm{O}_{3}$ (PZT) or $\mathrm{Pb}\left(\mathrm{Mg}_{1 / 3} \mathrm{Nb}_{2 / 3}\right) \mathrm{O}_{3}-(30$ at. $\%) \mathrm{PbTiO}_{3}$ (PMN-PT) piezoelectric layer(s) laminated together with magnetostrictive Terfenol-D ones operated under electromechanical resonance (EMR) conditions.

\section{MAGNETOELECTRIC LAMINATE COMPOSITES AS GYRATORS}

In Fig. 1(b), we illustrate a ME laminate that has a transversely poled piezoelectric layer sandwiched between two longitudinally magnetized Terfenol-D ones, i.e., a longitudinal-transverse (L-T) mode configuration. ${ }^{6}$ Our investigations were not limited to this mode; rather we only show it as an illustration. We have also studied laminates with longitudinally magnetized Terfenol-D and longitudinally poled piezoelectric layers [or longitudinal-longitudinal (L-L) mode], and a "push-pull" configuration that is a L-L mode whose piezoelectric layer is symmetrically poled. ${ }^{7}$ The piezoelectric PZT layers were polycrystalline, whereas the PMN-PT ones were (001) oriented crystals. Details concerning composite fabrication can be found in other recent publications concerning ME effects. ${ }^{4-7}$

A ME laminate with a coil wrapped around it is a fourpole device. In this case, the impedances of (1), where the subscript 1 refers to the piezocircuit and the subscript 2 refers to the coil circuit, are given as

$$
\begin{aligned}
& Z_{11}=-i \frac{1}{2 \pi f} \frac{d}{\varepsilon_{\mathrm{EFF}} l w}, \quad Z_{12}=N \frac{\alpha_{\mathrm{ME}}}{\varepsilon_{\mathrm{EFF}}} \frac{d}{l}, \\
& Z_{21}=-N \frac{\mu_{\mathrm{EFF}}}{\alpha_{\mathrm{ME}}} \frac{d}{l}, \quad Z_{22}=i 2 \pi f \frac{N^{2} d w \mu_{\mathrm{EFF}}}{l},
\end{aligned}
$$

where $Z_{11}$ and $Z_{22}$ are the impedances of the piezoelectric layer(s) and coils, respectively; $Z_{12}$ and $Z_{21}$ are equal to a gyrator coefficient (designated as $\alpha$ ); $d, l$, and $w$ are the thickness, length, and width of the ME laminate; $N$ is the number of the coil; $f$ is the frequency; and $\varepsilon_{\text {eff }}, \mu_{\text {eff }}$, and $\alpha_{\mathrm{ME}}$ are the effective dielectric, permeability, and ME susceptibilities of the laminate. An ideal (or Tellegen) gyrator has the following additional imposed restriction ${ }^{1}$ between $\varepsilon_{\text {eff }}, \mu_{\text {eff }}$ and $\alpha_{\mathrm{ME}}$ :

$$
\frac{\alpha_{\mathrm{ME}}}{\sqrt{\mu_{\mathrm{EFF}} \varepsilon_{\mathrm{EFF}}}} \approx 1,
$$

which simplifies (3) to

$$
\begin{aligned}
& Z_{12}=-Z_{21}, \\
& Z_{11} Z_{22}=-Z_{12} Z_{21} .
\end{aligned}
$$

We find for ME laminates, compared to these two criteria for ideal gyrators, that (i) the first one of (5a), $Z_{12}=-Z_{21}$, is met, but (ii) the second one of (5b) is not met. Although we can change $Z_{12}$ and $Z_{21}$ by varying $Z_{11}$ and $Z_{22}$, at best we can achieve only either $Z_{11}$ or $Z_{22}$ close to zero.

\section{TESTING METHODS}

Measurements were then performed using a dual lock-in amplifier method to calibrate the phase difference of the two signals [i.e., that of both the piezo and magnetic sides of the circuit in Fig. 1(a)]. A de magnetic field of 500 Oe was applied along the length of Terfenol-D, and a 50 turn coil (tightly wound without gaps) was wrapped around the laminates. The experimental setup is shown in Fig. 1(c). When exciting the piezolayer(s), the output BNC connector $\left(10^{17} \Omega\right.$ resistance and $15 \mathrm{pF}$ capacitance, which can be considered as an open circuit) of the lock-in (SR850 DSP) was connected to a $1 \mathrm{k} \Omega$ resistor, which was connected to both sides of the piezolayer(s), and the input BNC connector $(50 \Omega)$ was connected in series with the drive coil.

The current of the coil $\left(I_{2}\right)$ was measured by connecting a small capacitor, via the voltage induced across the capacitor. The voltage of the coil $\left(V_{2}\right)$ was calculated by that applied from the lock-in, subtracting the resistance of the input BNC connector multiplied by $I_{2}$. The voltage induced across the piezolayer(s) was directly measured by the lock-in under open circuit conditions, and the induced current $\left(I_{1}\right)$ was measured using a small resistor that short circuited the piezolayer(s). During phase measurements, an ac voltage source was used to excite the coils and a small resistor was connected to the source and used as a reference signal for the lock-in. This reference signal had the same phase as the current of the exciting coil. The lock-in can then gave the phase shift $(\Delta \phi)$ for the detected signal relative to the reference one, yielding a measure of $\Delta \phi$ between the voltage induced across the piezolayer(s) $\left(V_{1}\right)$ and the current of the exciting coil $\left(I_{2}\right)$. We also measured the phase shift between the induced current of the piezolayer(s) $\left(I_{1}\right)$ and that of a drive voltage applied to the coils $\left(V_{2}\right)$.

\section{CONFIRMATION OF UNIDIRECTIONAL GYRATOR CHARACTERISTICS}

In Fig. 2, we present the frequency dependence of the phase shifts between the voltage and current, which was taken under different excitation conditions. Also, in the inset of Fig. 2, we show the dependence of the gyration coefficient on frequency. As can be seen in Fig. 2, the required phase difference between open and short circuit conditions was met over a broad frequency bandwidth of $1<f<10 \times 10^{5} \mathrm{~Hz}$; but as can be seen in the inset, the required condition for ideal gyration in (5a) was fulfilled only at the EMR condition. These results unambiguously demonstrate the existence of a $180^{\circ}$ phase shift between $I$ and $V$. This is the report of such a $180^{\circ}$ phase shift at low frequencies (less than gighertz), and it is distinctly different than the conventional $90^{\circ}$ shift between $I$ and $V$ as in the case of usual reactive elements of the circuit (i.e., $L$ or $C$ ). At the resonance frequency, the phase of PZT changed rapidly, which caused the phase shift between $V_{2}$ and $I_{1}$ and between $V_{1}$ and $I_{2}$ to change rapidly near the resonance frequency. The results in Fig. 2 establish (i) the nonreciprocal nature of the couple and (ii) the nondissipative nature of the $I-V$ conversion, i.e., current is not generated by a voltage drop at the EMR. 


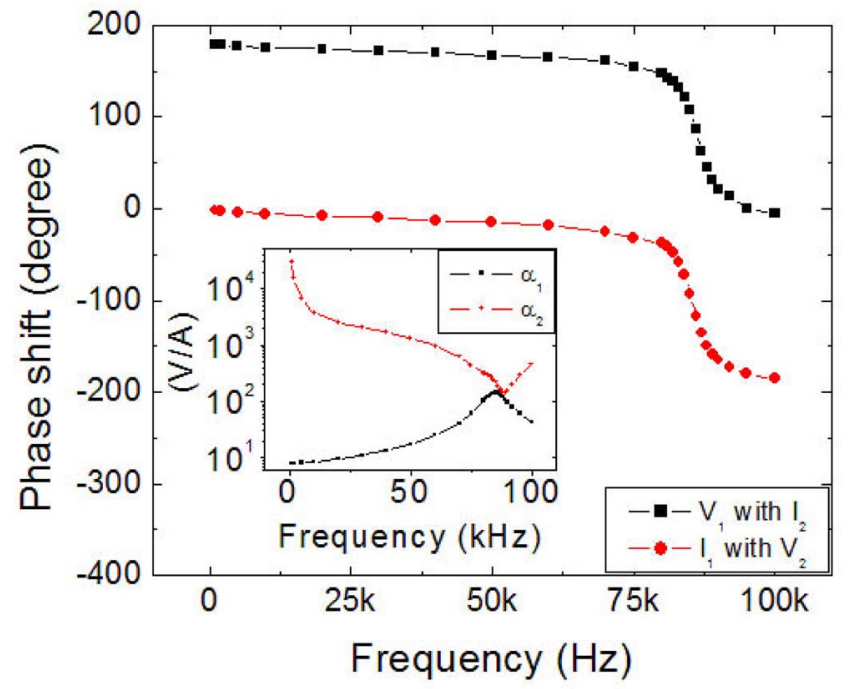

FIG. 2. (Color online) Phase difference between open and short circuit conditions for a L-T mode PZT/Terfenol-D laminate as a function of frequency from 1 to $10^{5} \mathrm{~Hz}$, where 1 designates the PZT capacitance side of the gyrator and 2 the inductance side of the coils. he inset shows the modulus of the gyration coefficient.

Figure 3 shows the impedance inverter property of our gyrator, given by (5b). The inset is an illustration of the measurement circuit. Here, $V$ is the voltage source, $R_{1}$ the output impedance of the voltage source, and $R_{2}$ a resistance of $2000 \Omega$ that is used to avoid possible open circuit conditions on the piezolayer side. First, we connected $Z$ in series to the piezolayers and measured the voltage induced on $R_{2}$. Then, we removed $Z$ and connected $Z^{\prime}$ in parallel to the coil, selecting a suitable value to let the induced voltage on $R_{2}$ be equal to that when it was connected in parallel with $Z$. The value of $\alpha$ was estimated as that of $\alpha 1$ and $\alpha 2$, as given in the inset of Fig. 2. The data in Fig. 3 were measured using the following values of resistances: $Z_{\text {piezo }}=2010 \Omega, Z_{\text {coil }}$ $=9.8 \Omega$, and $\alpha=150 \Omega$ (all at a resonance frequency of $84 \mathrm{kHz}$ ). As can be seen in Fig. 3, our construction exhibited good characteristics as an impedance inverter. Although changing $R_{1}$ or $R_{2}$ changed $\alpha$, we obtained a linear relation between $Z$ and $1 / Z^{\prime}$. If $Z$ and $Z^{\prime}$ were changed to a capacitor and an inductor (but not a pure resistor), similar results were obtained except that there was about a $15^{\circ}$ phase change introduced. These findings show that the ME gyrator is like an ideal gyrator, but one in reality connected to a capacitor on one side and an inductor on the other. In the future, by use of other ME laminate configurations, we hope to decrease

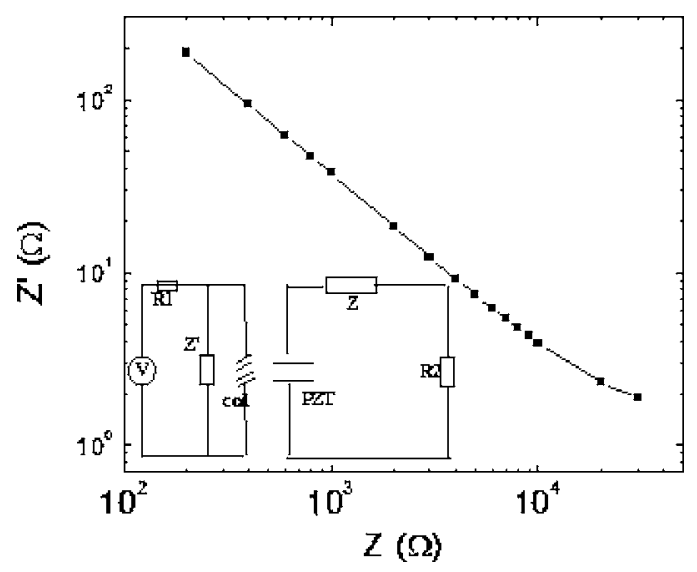

FIG. 3. Impedance inverter property of our gyrator under different resistance loads. The inset shows the measurement circuit. $Z$ and $Z^{\prime}$ are not coexisting. Measurements were done near a resonance frequency of $84 \mathrm{kHz}$.

the values of the resistances $Z_{\text {piezo }}$ and $Z_{\text {coil }}$ simultaneously while at the same time maintaining the same high value for the gyration coefficient.

\section{SUMMARY}

Tellegen suggested a bidirectional ideal gyrator; however, we have found that ME laminates are unidirectional gyrators with good gyration characteristics. Accordingly, our four-pole device is a small, discrete, passive network element that offers a type of electrical component capable of tuning stray or mutual inductances in a circuit into purely capacitive ones. As a fundamental network element, it could offer considerably improved and/or simplified solutions to many complex network problems. We have found, while at the EMR, that ME laminates have high gyration. In summary, our findings indicate the potential existence of a fifth fundamental network element.

\section{ACKNOWLEDGMENT}

This work was supported in full by the Office of Naval Research.

${ }^{1}$ B. D. H. Tellegen, Philips Res. Rep. 3, 81 (1948).

${ }^{2}$ C. Hogan, Rev. Mod. Phys. 25, 253 (1953).

${ }^{3}$ G. Smolenskii and I. Chupis, Sov. Phys. Usp. 25, 475 (1982).

${ }^{4}$ M. I. Bichurin, V. M. Petrov, and G. Srinivasan, J. Appl. Phys. 92, 7681 (2002).

${ }^{5}$ M. I. Bichurin, V. M. Petrov, and G. Srinivasan, Phys. Rev. B 68, 054402 (2003).

${ }^{6} \mathrm{~S}$. Dong, J. Li, and D. Viehland, IEEE Trans. Ultrason. Ferroelectr. Freq. Control 51, 794 (2004).

${ }^{7}$ S. Dong, J. Li, and D. Viehland, Appl. Phys. Lett. 83, 4812 (2003).

${ }^{8}$ S. Dong, J. Li, and D. Viehland, J. Appl. Phys. 95, 2625 (2004). 